\title{
Janus Colloids Actively Rotating on the Surface of Water
}

\author{
Xiaolu Wang, ${ }^{\dagger}$ Martin In, ${ }^{\dagger}$ Christophe Blanc, ${ }^{\dagger}$ Alois Würger, ${ }^{\ddagger}$ Maurizio Nobili, ${ }^{\dagger}$ and Antonio Stocco ${ }^{* \dagger \odot}$ \\ ${ }^{\dagger}$ Laboratoire Charles Coulomb (L2C), Université de Montpellier, CNRS, 34095 Montpellier, France \\ ${ }^{\ddagger}$ Laboratoire Ondes et Matière d’Aquitaine, Université de Bordeaux, CNRS, 33405 Talence, France
}

ABSTRACT: Biological or artificial microswimmers move performing trajectories of different kinds such as rectilinear, circular, or spiral ones. Here, we report on circular trajectories observed for active Janus colloids trapped at the air-water interface. Circular motion is due to asymmetric and nonuni form surface properties of the particles caused by fabrication. Motion persistence is enhanced by the partial wetted state of the Janus particles actively moving in two dimensions at the air-water interface. The slowing down of in plane and out of plane rotational diffusions is described and discussed.
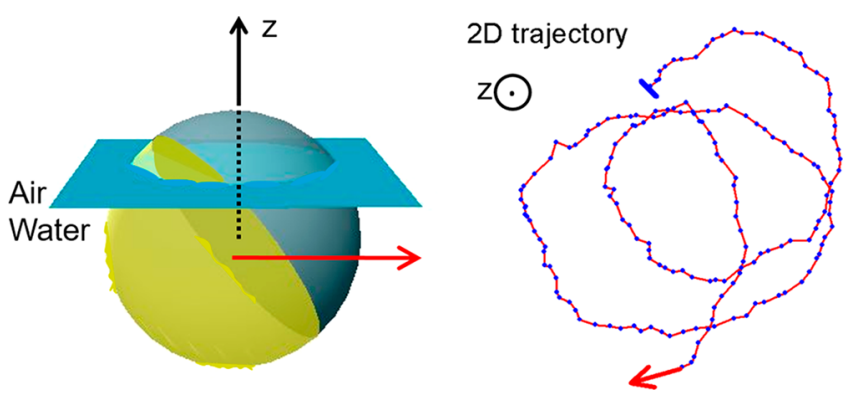

\section{INTRODUCTION}

Microswimmers (biological or artificial active particles) often move in confined geometries such as close to surfaces, membranes or inside a compartment. ${ }^{1,2}$ Motion trajectory results from the shape of the particle, the propulsion mechanism, and the confinement. ${ }^{2,3}$ Recently, artificial active particles confined close to a solid wall or trapped at air-liquid or liquid-liquid interfaces have been investigated. ${ }^{4-7}$ In these confinement geometries, the motion becomes purely two dimensional. $^{7}$ The most popular class of artificial active particles investigated so far is Janus colloids. ${ }^{3,8}$ Such particles are made of two distinct regions with quite different properties. The regional disparity in surface properties implemented in Janus colloids may concern shape, surface charge, roughness, or chemical activity. ${ }^{9}$ The large choice in the design of Janus colloids opened new routes in many fields including self assembly $^{10}$ and wetting, ${ }^{11}$ and in the field of micromachines, which are systems able to perform specific tasks such as transporting cargos or cleaning water. ${ }^{12}$

A striking property of Janus colloids consists of their ability to move autonomously and explore defined regions of space. ${ }^{13,14}$ The autonomous motion of Janus colloids is usually implemented by coating one particle region with a nanometric platinum layer, which transforms the chemical fuel $\mathrm{H}_{2} \mathrm{O}_{2}$ added in the environment into water and oxygen, and gives rise to a self phoretic propulsion of the colloid. ${ }^{15,16}$ Roughness, ${ }^{17}$ thickness, ${ }^{16,18}$ and shape ${ }^{19}$ of the platinum coating are important parameters which not only strongly affect the propulsion velocity $V$ but can also lead to an angular velocity $\omega$ of the colloid even if the original particle shape is spherical. ${ }^{20}$ In a recent paper, Archer et al. were able to control the rotational propulsion of spherical Janus colloids in the bulk. ${ }^{20}$ Such Janus colloids were fabricated by a glancing angle deposition technique. ${ }^{21}$ Changing the glancing angle of the platinum metal evaporation leads to asymmetric shapes of the coating with different covered areas. At normal glancing angle $\left(\Theta=90^{\circ}\right)$, a certain variability $0<\omega / \mathrm{rad}_{\mathrm{s}}{ }^{-1}<2$ of the angular velocity was observed in the bulk. By reducing the glancing angle to $\Theta=20^{\circ}, \omega$ increases up to $18 \mathrm{rad} . \mathrm{s}^{-1}$ and the projected trajectories observed were essentially circular. Note that similar circular or spiral trajectories were observed previously for strongly asymmetric particles or for spherical light adsorbing particles under optical fields. ${ }^{3,22-26}$

Roughness, shape, and covered area of the platinum coating also affect the wetting properties of Janus colloids trapped at an air-water interface, as we have recently shown for the Brownian and active motion of platinum-silica Janus colloids confined at the surface of water. ${ }^{4,27}$ Their active motion was mostly characterized by rectilinear trajectories with a motion persistence significantly enhanced when compared to the active motion in bulk. ${ }^{4}$ The impact of the wetting dynamic on the rotational Brownian motion of Janus particles was also discussed recently by us. ${ }^{27}$ The severe slowing down of the rotational diffusion about an axis perpendicular to the interface normal $D_{\mathrm{r}, \perp}$, which is characteristic of partial wetting, contributes to the enhancement in the motion persistence. ${ }^{4,27}$

In this article, we focus on the rotational active motion and rotational diffusion $D_{\mathrm{r}, \|}$ about an axis parallel to the interfacial normal. As already observed in the bulk, ${ }^{15,20}$ a variability in the fabrication of spherical Janus particle can lead to a persistent angular velocity. Here, by analyzing in great detail the active rotation of Janus colloids at the air-water interface, we were able to evaluate both the contributions of the random and the active motion to the in plane orientation dynamics of the Janus colloids.

\section{EXPERIMENTAL SECTION}

The Janus colloids used in this paper are spherical silica beads $\left(\mathrm{SiO}_{2}\right.$, purchased from Microparticles $\mathrm{GmbH}$, radius $R=1.06 \pm 0.03 \mu \mathrm{m}$ ) half coated by a platinum layer. They have been prepared according to 

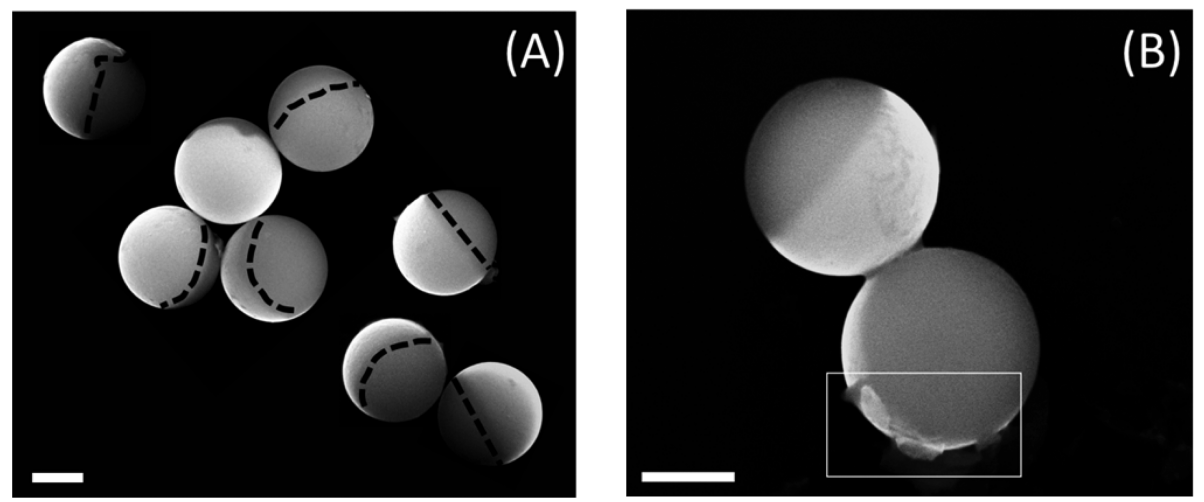

Figure 1. (A) SEM images of Janus particles. Dashed lines show the Janus boundaries. (B) SEM image of two Janus particles, where surface defects are visible (see inside the white box). Scale bar is $1 \mu \mathrm{m}$.
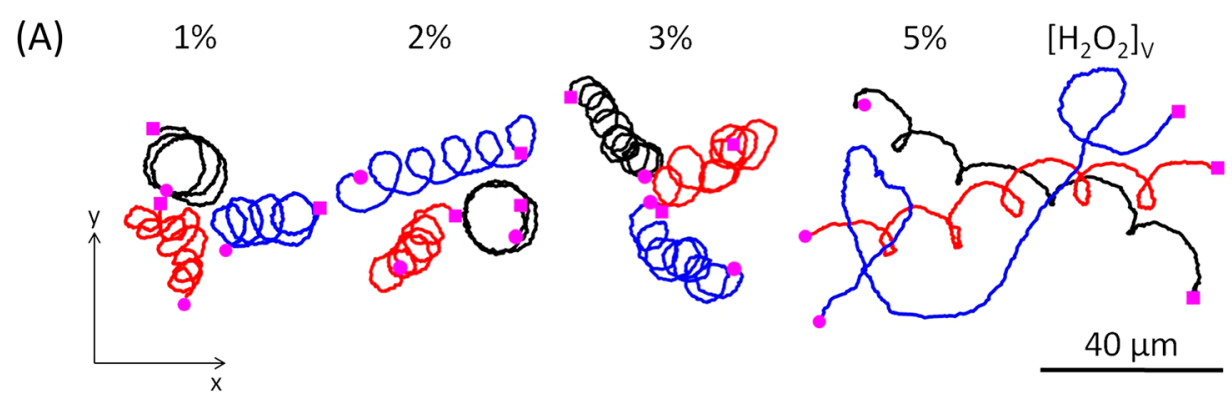

(B)
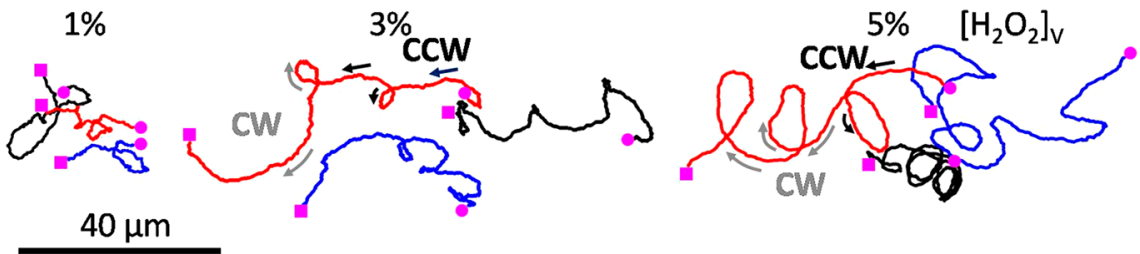

Figure 2. (A) Selected circular trajectories of Janus particles at the horizontal air-water interface $(x y$ plane $)$ under different fuel concentrations $\left(\left[\mathrm{H}_{2} \mathrm{O}_{2}\right]_{\mathrm{V}}\right)$ over $20 \mathrm{~s}$. The filled circle represents the beginning of the motion and the filled square is the end. (B) Two dimensional projections of three dimensional trajectories performed by Janus particles moving in bulk water under different fuel concentrations $\left(\left[\mathrm{H}_{2} \mathrm{O}_{2}\right]_{\mathrm{V}}\right)$ over $15 \mathrm{~s}$. The filled circle represents the beginning of the motion and the filled square is the end. The arrows represent rotational directions. There are apparent switches between CCW and CW rotations represented by the red curves at 3\% and $5 \%$ fuel concentrations.

the method described by Love et al., ${ }^{28}$ with some modifications. ${ }^{4,27}$ Particle fabrication and characterization were already described in our previous articles. ${ }^{4,27}$ Briefly, we start by preparing a monolayer of silica beads. Then, using a plasma bombarded metal sputtering (Alcatel SCM 400 system), first $10 \mathrm{~nm}$ of titanium and then $20 \mathrm{~nm}$ platinum are coated onto the top of silica bead surfaces. Such Pt coated silica particles present variability in the coating structure, which is intrinsic to the fabrication protocol. ${ }^{27}$ The Janus boundaries show some "linear" or "wavy" shapes (see Figure 1), and the Pt layer presents roughness at a characteristic length scale of about $10 \mathrm{~nm}$, which corresponds to the height and the lateral dimension of the platinum patches. ${ }^{27}$ Janus boundaries may also present larger defects as shown in Figure 1B. In order to investigate the motion of Janus colloids at the surface of water, a water suspension of particles was sprayed onto a bare water surface. ${ }^{4}$ The motion of isolated Janus colloids at the surface of water and in the water bulk were both tracked by CCD camera equipped microscopes, and then the videos were analyzed using the Labview Stat Tracker St. Andrews software to obtain the corresponding trajectories of particles [time $t(\mathrm{~s}), x(\mu \mathrm{m}), y(\mu \mathrm{m})]^{4}$ where the $x$ and $y$ axes define the horizontal planes in the fixed laboratory coordinate system. The Pt coated areas of particles tracked could also be distinguished from the bare silica surfaces after treating the videos by imputing thresholds to the raw images using the commercial software $I D L \mathrm{v}$ 5.4.

\section{RESULTS AND DISCUSSION}

Catalytic Janus colloids were irreversibly attached onto the airwater interface, with a contact angle $\alpha$ of about $57^{\circ}$ : the advancing contact angle $\alpha_{\mathrm{A}}=64 \pm 2^{\circ}$ was measured by a gel trapping method and receding contact angle $\alpha_{\mathrm{R}}=50 \pm 6^{\circ}$ by optical microscopy. ${ }^{27}$ These particles perform autonomous active motion in the presence of $\mathrm{H}_{2} \mathrm{O}_{2} \cdot{ }^{4}$ Recently, we reported on the active motion of Janus colloids at the air-water interface showing rectilinear like trajectories with an enhanced persis tence length when compared to the trajectories observed in bulk. $^{4}$

In the following, we describe motion features of active circular like trajectories, which are related to the nonideal properties of Janus particles. Two quite different aspects of how surface imperfections affect the particle motion are also discussed. The first one concerns rotational motion due to static inhomogeneities of the particle surface, and the second one concerns velocity fluctuations due to jumps of the contact line between metastable pinning states.

Circular Trajectories at the Surface of Water and in Bulk. In this paper, we report some experiments showing 
circular trajectories at the air-water interface (see Figure 2A). Using the quantitative but arbitrary criteria discussed in the next paragraph, we found that these trajectories represent $28 \%$ of the active trajectories observed at the air-water interface. Circular trajectories were also observed in the bulk far from the solid interface of the bottom of the container (see Figure 2B). Hence, the circular particle motion can be safely attributed to the particle fabrication which can cause a break of symmetry in the particle geometry. We could attempt to compare particle surface defects observed in SEM images with the kind of trajectories observed, i.e., circular or rectilinear. About $40 \%$ of the particles show either wavy Janus boundaries (Figure 1A) or some asymmetric platinum coating (Figure 1B). However, this comparison should be considered only qualitative since it is difficult to establish quantitative criteria on the critical size or shape of the surface defects which would lead to a break of symmetry in the particle geometry and to the observation of circular motion.

As shown in the next section, we calculated the propulsion velocity $V$ and angular velocity $\omega$ for each trajectory. Arbitrarily, we choose the criterion $V / \omega<25 \mu \mathrm{m}$ to distinguish between circular trajectories and rectilinear trajectories. ${ }^{4}$ For $V / \omega<25$ $\mu \mathrm{m}$, circular trajectories are easy to identify, as shown in Figure 2. When the propulsion speed $V$ is high or the angular frequency is low and $V / \omega>25 \mu \mathrm{m}$, it is difficult to evaluate $\omega$. In Figure 3, we show the distribution of $V / \omega$ for the

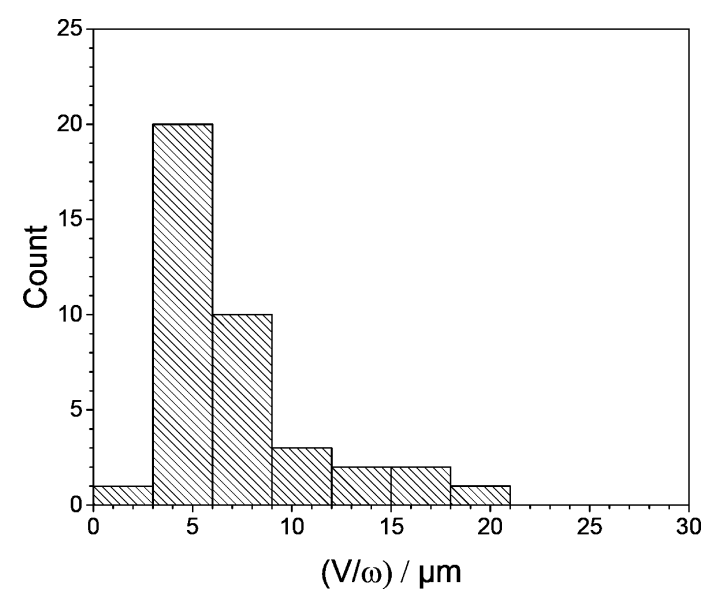

Figure 3. Distribution of the experiments showing circular trajectories characterized by the ratio between the linear propulsion velocity and the angular velocity, i.e., $V / \omega$.

experiments at the air-water interface described in this paper. $V / \omega$ distribution shows the highest values between 3 and $9 \mu \mathrm{m}$, which can be related to the radius of curvature of the trajectories observed in Figure 2. At the air-water interface, in the experimental time window of $20 \mathrm{~s}$ and for each measurement, the rotation direction of the trajectory always remains the same. We observed both clockwise (CW) and counterclockwise $(\mathrm{CCW})$ rotation in all the different experi ments and for different beads. Note, however, that for each trajectory, we never observed a switch of the rotation direction during the experiment. In the bulk far from the solid interface, in contrast, we observed that the rotational direction of the projected three dimensional trajectories can switch during the active motion (see Figure 2).

Ideally symmetrical and spherical Janus colloids are expected to move actively along a straight line, because of the symmetry plane formed by its axis and the $z$ axis (see Figure 4A). For nonideal Janus colloids as in Figure $4 \mathrm{~b}$, however, this symmetry

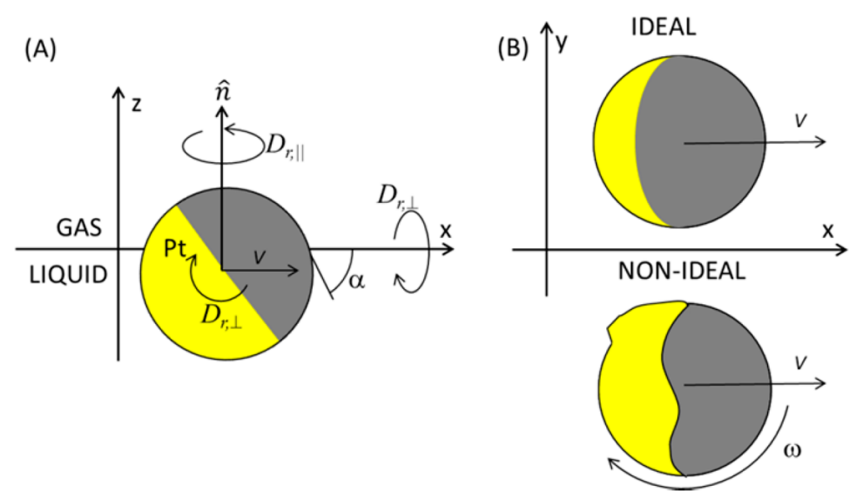

Figure 4. Sketches: (A) Side view of an ideal Janus colloid attached onto a gas-liquid interface with a contact angle $\alpha$. (B) Top view of an ideal and nonideal Janus colloid and related effective linear propulsion speed $V$ and angular velocity $\omega$.

is broken by the nonuniform surface properties. As a consequence, besides the linear propulsion velocity $V$, there is a finite angular velocity $\omega$ about the $z$ axis (see Figure 4B). ${ }^{29}$ At the interface, the rotation of a Janus colloid about an axis in the interfacial plane ( $x$ or $y$ axis) involves a relative motion between the liquid and the solid surface of the colloid as in partial wetting of drops on planar surfaces (see Figure 4A). Upon an out of plane rotation at the interface, the dry region of the colloid surface will wet and the wetted region will dewet. These phenomena involve high dissipations and a characteristic friction related to contact line motion over surface defects. Recently, we have shown that the characteristic time of the out of plane rotational motion could be as high as $1 / D_{\mathrm{r}, \perp}=152 \mathrm{~s} \gg$ $1 / D_{\mathrm{r} \text { bulk. }}{ }^{27}$ Hence the absence of switching between rotation directions at the interface shown in Figure 2 is a clear manifestation of the severe slowing down of the out of plane rotational diffusion $D_{\mathrm{r}, \perp}$, which prevents the rotation of the colloid about the motion direction axis.

Diffusions and Velocities Obtained by Image Analysis and Trajectory Data. For some recorded trajectories, it was possible to detect the orientation of the Janus particle within the interfacial plane and to determine its rotational diffusion coefficient. Since we did not use fluorescent particles, the analysis relied on binarizing in black and white the gray level of the image. ${ }^{30}$ When this allowed to clearly distinguished between the platinum covered area and bare surface area, then the orientation could be measured as the angle $\varphi=\arctan \left(\frac{y_{0}-y_{1}}{x_{0}-x_{1}}\right)$, where $\left(x_{1}, y_{1}\right)$ are the coordinates of the center of the platinum coated area and $\left(x_{0}, y_{0}\right)$ of the center of the whole particle (see Figure 5). The success of the procedure relied not only on the particle orientation and illumination conditions to get enough primary contrast but also on distance between both points relative to optical resolution.

For the experiments showing significant values of the distance between the center of the particle and the platinum coated area (see Figure 5), we calculated the mean squared angular displacement $\mathrm{MSAD},\left\langle\Delta \varphi^{2}\right\rangle$ (see Figure 6).

MSAD data were fitted by ${ }^{31}$

$$
\left\langle\Delta \varphi^{2}\right\rangle=2 D_{\mathrm{r}, \|} \Delta t+w^{2} \Delta t^{2}
$$




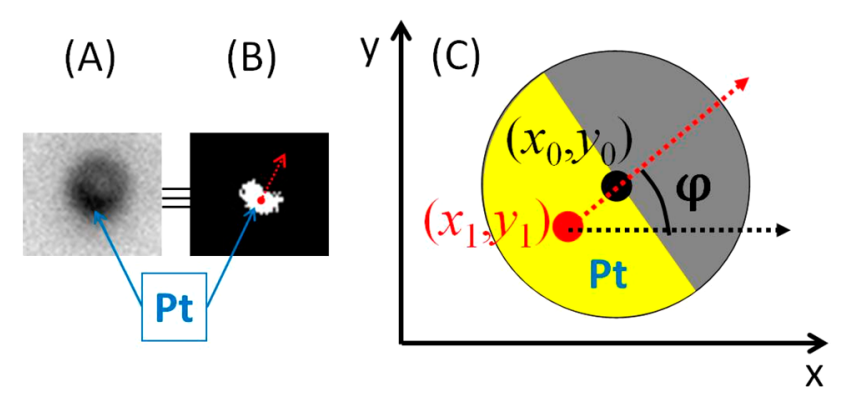

Figure 5. (A) Raw image of Janus colloid from which the position $\left(x_{0}\right.$, $y_{0}$ ) of the center of the whole particle is determined. (B) Thresholded image from which the position $\left(x_{1}, y_{1}\right)$ of the center of the platinum coated area is determined. (C) Orientation of the Janus particle is characterized by the angle $\varphi$.

in order to find the in plane rotational diffusion coefficient $D_{\mathrm{r}, \|}$ and the absolute value of the angular velocity modulus $\omega$. Equation 1 was obtained from a two dimensional Langevin description of the active motion where the propulsion velocity, angular velocity, and rotational diffusion are assumed to be constants and decoupled from each other. ${ }^{31}$

Note that MSAD is linear with the lag time for passive Brownian particles. At short times, one sees the contributions of the linear and quadratic terms for active colloids and MSAD data deviates from a purely quadratic form as shown in Figure 6B. $D_{\mathrm{r}, \|}$ and the calculated bulk rotational diffusion coefficient $D_{\mathrm{r}, \text { bulk }}=\frac{k T}{8 \pi \eta R^{3}}$ (where $k T$ is the thermal energy, $\eta$ is the fluid viscosity, and $R$ is the particle radius) as a function of the angular velocity are plotted in Figure 7 . We also did some measurements in the absence of $\mathrm{H}_{2} \mathrm{O}_{2}$ fuel where we observed simple Brownian motion $(\omega=0)$. For $\omega<1.5 \mathrm{rad} . \mathrm{s}^{-1}, D_{\mathrm{r}, \|}$ is lower than the bulk diffusion coefficient by a factor 2 to 5 . For $\omega>1.5$ rad.s ${ }^{-1}, D_{\mathrm{r}, \|}$ increases and becomes about $1.5 \times D_{\mathrm{r}, \mathrm{bulk}}$. Note that for ideal spherical bare particles half immersed in a liquid (and half in air), the expected in plane rotational diffusion coefficient is $D_{\mathrm{r}, \|}=2 D_{\mathrm{r}, \mathrm{bulk}}$ in no slip conditions, assuming a flat fluid interface and no effect of the three phase contact line fluctuations. ${ }^{32}$ The slowing down of the rotational diffusion for passive Janus particles $(\omega=0)$ agrees with measurements recently done on low aspect ratio ellipsoidal particles. ${ }^{33,34}$ More detailed results on passive Janus particles will be given in a forthcoming paper in which the effect of

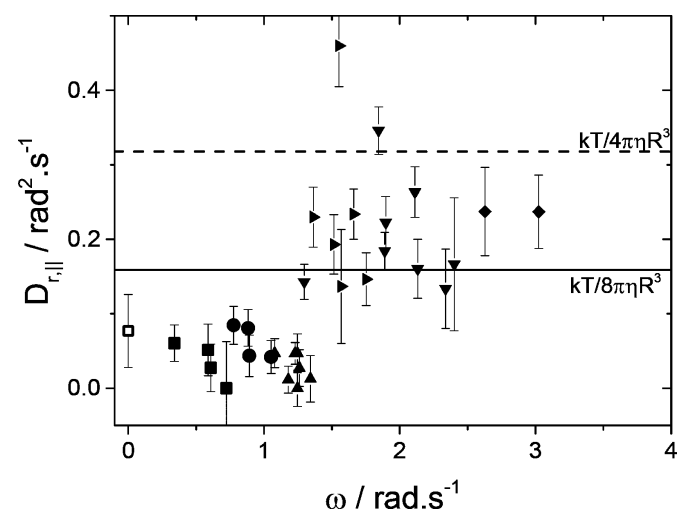

Figure 7. Rotational diffusion coefficients about the interface normal as a function of the particle angular velocity. The point at $\omega=0$ corresponds to the measurement of Brownian Janus colloid in the absence of $\mathrm{H}_{2} \mathrm{O}_{2}$. Different symbols correspond to different particles and different points are obtained at different times. The solid and dashed lines respectively represent the theoretical bulk rotational diffusion and the derived rotational diffusion coefficient about the interface normal for an half immersed particle.

partial wetting on the rotational and translational motion will be described.

Complementary to the image analysis, we also studied the trajectories of the active particles and calculated the discrete velocity $\mathbf{v}$, which has two components in the $x$ and $y$ laboratory axis, and modulus $|v|=\sqrt{v_{x}^{2}+v_{y}^{2}}$, where $v_{x}(t)=\frac{x\left(t+\Delta t_{1}\right)-x(t)}{\Delta t_{1}}$ and $v_{y}(t)=\frac{y\left(t+\Delta t_{1}\right)-y(t)}{\Delta t_{1}}$ and $\Delta t_{1}=1 / 30 \mathrm{~s}$ is the lowest time interval dictated by our CCD camera.

Discrete velocity autocorrelation functions $\langle\mathbf{v}(t+\Delta t) \mathbf{v}(t)\rangle=$ $\left\langle\mathbf{v}_{x}(t+\Delta t) \mathbf{v}_{x}(t)\right\rangle+\left\langle\mathbf{v}_{y}(t+\Delta t) \mathbf{v}_{y}(t)\right\rangle$ for different angular velocities $\omega$ are plotted in Figure 8.

In a purely two dimensional system, for active colloids showing both a propulsion velocity $V$ and an angular velocity modulus $\omega$, the autocorrelation function of the instantaneous velocity $\mathbf{v}_{i}$ reads: ${ }^{31}$

$$
\begin{aligned}
& \left\langle\mathbf{v}_{\mathbf{i}}(t+\Delta t) \cdot \mathbf{v}_{\mathbf{i}}(t)\right\rangle=4 D_{\mathrm{t}, 2 D} \delta(\Delta t)+V^{2} \cos (\omega \Delta t) \\
& \quad \exp \left(-D_{\mathrm{r}, 2 D} \Delta t\right)
\end{aligned}
$$
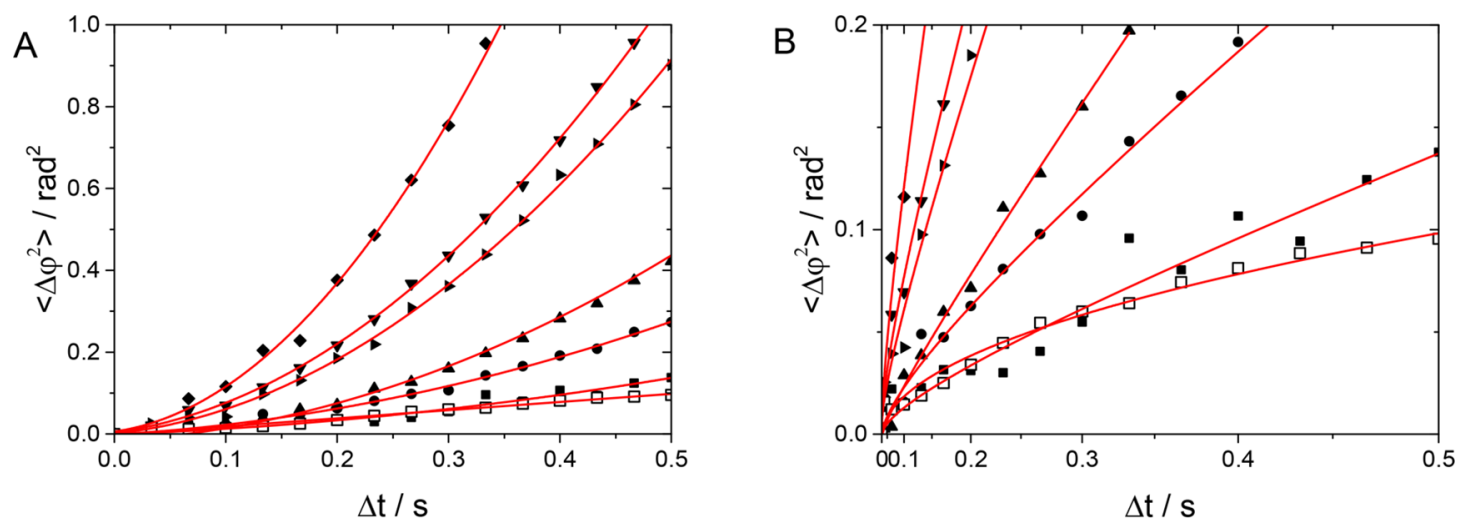

Figure 6. Mean squared angular displacement at the air-water interface for a passive (open square symbols) and active Janus colloid of different angular velocities (filled symbols) plotted as a function of the lag time in (A) linear scale and (B) quadratic scale (with a zoom in the vertical axis). Different symbols correspond to different particles. Solid lines are the fits to the data points using eq 1. 


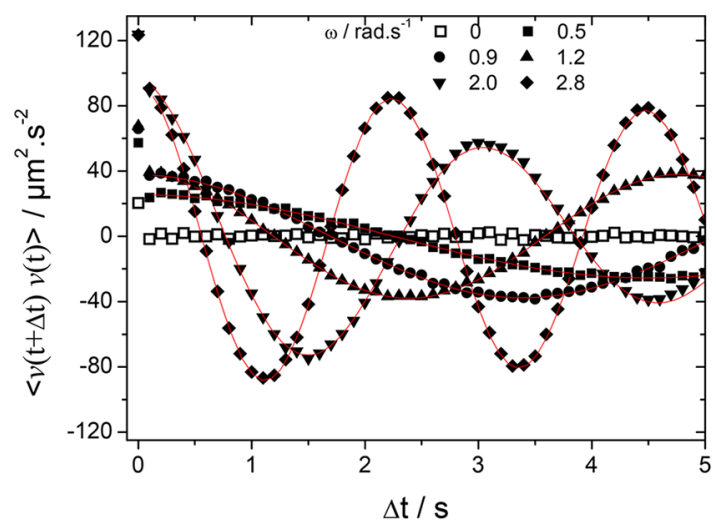

Figure 8. Velocity autocorrelation function $\langle\mathbf{v}(t+\Delta t) \mathbf{v}(t)\rangle$ as a function of the lag time $\Delta t$ for the same particles shown in Figure 6. $\omega$ in the legend are the average values obtained from MSAD analysis. Solid lines are the fits to the data points using eq 4 .

where $\delta(\Delta t)$ is the Dirac distribution. The first term is related to the $2 \mathrm{D}$ translational Brownian diffusion that is present for both passive and active colloids. The second term accounts for the active motion and the change of motion persistence due to the Brownian rotation.

Given the severe slowing down of out of plane rotation, such an equation should still hold in our system with $D_{\mathrm{t}, 2 \mathrm{D}}=D_{\mathrm{t}}$ (the translational diffusion coefficient at the interface of our system) and $D_{\mathrm{r}, 2 \mathrm{D}}=D_{\mathrm{r}, \|}$, already introduced. Hence, we used eq 2 to extract the velocities and diffusions from our experimental data (Figure 8).

First, note that for passive colloids $(V=0)$, the diffusion coefficient $D_{\mathrm{t}}$ can be extracted from the particle discrete velocity autocorrelation function at $\Delta t=0 \mathrm{~s}$, since ${ }^{35}$

$$
v^{2}=\Delta r^{2} / \Delta t_{1}^{2}=4 D_{t} / \Delta t_{1}
$$

In Figure 8, for both passive and active measurements at the air-water interface, discrete autocorrelation functions show a clear step between $\Delta t=0$ and $\Delta t=\Delta t_{1}=1 / 30 \mathrm{~s}$, which is connected to the first term of eq 2 and eq 3 . For passive measurements, the autocorrelation drops to zero, since $\langle\mathbf{v}(t+$ $\Delta t) \cdot \mathbf{v}(t)\rangle=0$ for $\Delta t>\Delta t_{1}{ }^{36}$ In the case of active colloids, at $\Delta t_{1}$ the autocorrelation does not drop to zero but instead gives the square of the propulsion speed, $\left\langle\mathbf{v}\left(t+\Delta t_{1}\right) \cdot \mathbf{v}(t)\right\rangle \approx V^{2}$. Since $\langle\mathbf{v}(t) \cdot \mathbf{v}(t)\rangle=4 D_{\mathrm{t}} / \Delta t_{1}+V^{2}, D_{\mathrm{t}}$ can be obtained from $D_{\mathrm{t}} \approx$
$1 / 4\left[\langle\mathbf{v}(t) \mathbf{v}(t)\rangle-\left\langle\mathbf{v}\left(t+\Delta t_{1}\right) \mathbf{v}(t)\right\rangle\right] \Delta t_{1} \quad\left(D_{\mathrm{t}}\right.$ could be alternately measured by fitting mean squared displacement MSD data in the short time limit, MSD $=4 D_{\mathrm{t}} \Delta t+V^{2} \Delta t^{2}$. Both methods provide very similar $D_{\mathrm{t}}$ within about $6 \%$ difference). In Figure 9, we plot the ratio between $D_{\mathrm{t}}$ and the calculated diffusion coefficient in the bulk $D_{\mathrm{t}, \text { bulk }}=\frac{k T}{6 \pi \eta R}$ as a function of the angular velocity modulus. The ratio $D_{t} / D_{t, b u l k}$ holds approximately constant in the $\omega$ range where $D_{\mathrm{r}, \|}$ increases (see Figure 7). The values obtained here agree with the results reported by Boniello et al. for passive bare colloids. ${ }^{33,34}$

Data shown in Figure 8 were fitted using the second term of eq 2 adding a term accounting for a constant drift velocity $V_{\mathrm{d}}$. Note that drift is ubiquitous in diffusion experiments, in particular, at the interface due to advection flows.

$$
\langle\mathbf{v}(t+\Delta t) \cdot \mathbf{v}(t)\rangle=V^{2} \cos (\omega \Delta \mathrm{t}) \exp \left(-D_{\mathrm{r}, \|} \Delta t\right)+V_{\mathrm{d}}^{2}
$$

for $\Delta t>\Delta t_{1}$. The expected autocorrelation function is therefore an oscillating cosine function damped by the $\exp \left(-D_{\mathrm{r}, \|} \Delta t\right)$ term, which fits the data shown in Figure 8 in an excellent way.

In the presence of an oscillating function, one could clearly distinguish between $V$ and $V_{\mathrm{d}}$, with $V_{\mathrm{d}}^{2}$ being the mean value around which the velocity autocorrelation function oscillates. Values of the drift velocities measured here are comparable to the drifts previously reported for bare particles at different $\mathrm{H}_{2} \mathrm{O}_{2}$ concentrations: ${ }^{4} V_{\mathrm{d}}=1 \pm 1 \mu \mathrm{m} / \mathrm{s}$ for $\left[\mathrm{H}_{2} \mathrm{O}_{2}\right]_{\mathrm{V}}=1 \%, V_{\mathrm{d}}$ $=2 \pm 2 \mu \mathrm{m} / \mathrm{s}$ for $\left[\mathrm{H}_{2} \mathrm{O}_{2}\right]_{\mathrm{V}}=2 \%, V_{\mathrm{d}}=1 \pm 1 \mu \mathrm{m} / \mathrm{s}$ for $\left[\mathrm{H}_{2} \mathrm{O}_{2}\right]_{\mathrm{V}}$ $=3 \%$, and $V_{\mathrm{d}}=3 \pm 3 \mu \mathrm{m} / \mathrm{s}$ for $\left[\mathrm{H}_{2} \mathrm{O}_{2}\right]_{\mathrm{V}}=5 \%$ (see Figure 2). Note that in the lag time window where data can be safely fitted $(\Delta t<5 \mathrm{~s})$ due to the measurement statistics, it is not possible to observe a clear damping of $\langle\mathbf{v}(t+\Delta t) \cdot \mathbf{v}(t)\rangle$. Thus, these data cannot generally be used to measure $D_{\mathrm{r}, \|}$.

A good agreement is also found between the angular velocity $\omega$ shown in Figure 7 obtained by the MSAD analysis (orientation angle $\varphi$ obtained by image processing, Figure 5) and $\omega$ shown in Figure 9 by the velocity autocorrelation function.

In order to confirm the results obtained above, we analyzed all experiments showing circular trajectories even if the image quality was not high enough to find the orientation angle (see Figure 5). From the velocity autocorrelation functions, we obtained $D_{\mathrm{t}}, V$, and $\omega$ for different particles at the air-water interface for different $\mathrm{H}_{2} \mathrm{O}_{2}$ concentrations. In Figure 9, we plot
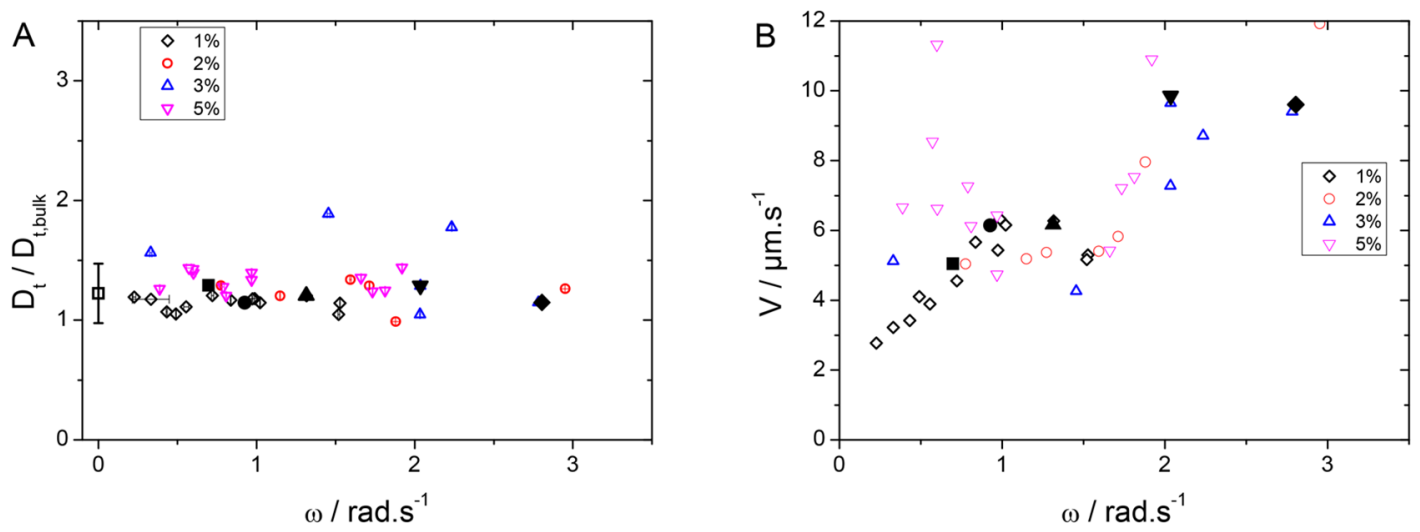

Figure 9. (A) Measurements of translational diffusion coefficients at the air-water interface divided by the calculated bulk diffusion coefficient and (B) propulsion velocity as a function of the angular velocity. Solid symbols correspond to the experiments shown in Figure 7. Open symbols correspond to all experiments showing circular trajectories where the orientation angle could be not evaluated. 
the data of particles with known orientation angle (full symbols) and those where the angle could not be determined (open symbols); the two sets show the same behavior.

Surface Inhomogeneities-Fluctuations of $\boldsymbol{V}$ and $\boldsymbol{\omega}$. The metal cap of Janus colloids is not a perfect hemisphere. Both its shape and its thickness show quite significant inhomogeneities, ${ }^{27}$ as illustrated in Figure 1. In addition, the chemical properties of both the silica and metal surfaces are not uniform but may vary from one spot to another. As a consequence, the catalytic activity and the interaction of the solute with the surfaces show inhomogeneities.

Here we discuss the distribution of the linear and angular velocities observed in Figure 9B. For a platinum coated Janus particle, the metal cap triggers chemical reactions which result in a nonuniform concentration $c$ of some molecular solutes (peroxide, oxygen, or some intermediate complex) which, in turn, gives rise to a slip velocity

$$
v_{\mathrm{s}}=\mu \nabla c
$$

where the mobility $\mu$ depends on the solute-surface interactions.

The slip velocity induces a complex flow profile in the particle's vicinity, and the corresponding viscous stresses at its surface determine the particle's self propulsion. For bulk particles, the stress integral is readily rewritten in terms of the surface averaged slip velocity. ${ }^{37}$ Since there is no such relation for interface particles, we adapt the well known expressions for bulk particles, and thus write for the linear velocity

$$
\mathbf{v}=-\oint \frac{\mathrm{d} S}{S_{0}}(1-\mathbf{n n}) \cdot \mathbf{v}_{\mathrm{s}}
$$

where $\mathbf{n}$ is the interface normal and $S_{0}$ the immerged fraction of the particle surface. The rotational motion about the $z$ axis is determined by the projected angular velocity vector ${ }^{37}$

$$
\omega=-\frac{3}{2 R} \oint \frac{\mathrm{d} S}{S_{0}} \mathbf{n n} \cdot\left(\mathbf{n}_{\mathrm{p}} \times \mathbf{v}_{\mathrm{s}}\right)
$$

with $\mathbf{n}_{\mathrm{p}}$ the normal on the particle surface. These equations are exact for a half immersed particle with its axis parallel to the interface. In the general case, the relations between slip velocity and particle motion are much more complex. In the following we assume that eqs 6 and 7 provide an approximate expression for the particle's linear and angular velocity.

The slip velocity field of an ideal Janus particle, $\mathbf{v}_{\mathrm{s}}=\mathbf{v}_{\mathrm{s}}^{0}=v_{\mathrm{s}}^{0} \mathbf{t}_{1}$ (where $t_{1}$ is a unit vector field on the particle) is symmetric with respect to the direction of motion; the particle then moves along a rectilinear trajectory with constant linear velocity and zero angular velocity. This behavior has indeed been observed at the air-water interface and described by Wang et al., ${ }^{4}$ and represents $72 \%$ of the entire active trajectories observed.

Some particles, however, show surface roughness, and the chemical activity and surface properties show significant deviations from the axisymmetric situation. As an obvious source we mention the inhomogeneous platinum cap illustrated in Figure 4, which results in a local change of the concentration profile $c(\mathbf{r})$. On the other hand, nonuniform chemical properties of the surfaces may also modify the mobility $\mu$ in eq 5 .

Thus, we write the slip velocity in the form

$$
\mathbf{v}_{\mathrm{s}}=v_{\mathrm{s}}^{0}\left[\mathbf{t}_{1}+\epsilon_{1} \mathbf{t}_{1}+\epsilon_{2} \mathbf{t}_{2}\right]
$$

where $\mathbf{v}_{\mathbf{s}}^{0}$ is the slip velocity of an ideal particle, $\mathbf{t}_{1}$ the unit tangent vector at the particle surface defined by the direction of the slip velocity $\mathbf{v}_{\mathbf{s}}^{0}$, and $\mathbf{t}_{2}$ the second unit tangent vector, perpendicular to $\mathbf{t}_{1}$. The additional terms arise from surface inhomogeneities, with position dependent factors $\epsilon_{i}(\mathbf{r})$ for the two tangent directions. Due to the individual variability, eqs 6 and 7 take finite values which are characteristic for the surface properties of each particle.

The statistical distribution of $V$ and $\omega$ is determined by the statistical properties of the factor $\epsilon_{i}(\mathbf{r})$. In the following, the mean velocity over an ensemble of particles is denoted $V$, and the root mean square deviation $\Delta V$. Regarding the angular velocity, we consider its vertical component $\omega$ only, which accounts for rotations about the interface normal. Its mean value vanishes, $\bar{\omega}=0$, such that its variance is identical to the second moment $\Delta \omega^{2}=\overline{\omega^{2}}$. Assuming that the $\epsilon_{i}(\mathbf{r})$ show the same statistical properties with zero mean, $\bar{\epsilon}_{i}=0$, and a mean square $\overline{\epsilon_{i}^{2}} \propto \epsilon^{2}$ given by the characteristic amplitude $\epsilon$, we find for the mean square deviation of linear and angular velocities,

$$
\Delta V=\sqrt{\frac{14}{15}} \epsilon v_{0} \quad \Delta \omega=\sqrt{\frac{9}{10}} \epsilon \frac{v_{0}}{R}
$$

Here we have assumed that the prefactor of the slip velocity varies with the polar angle $\theta$ with respect to the particle axis, $v_{\mathrm{s}}^{0}$ $=v_{0} \sin \theta$, like for a bulk particle; no such assumption is made concerning its direction $\mathbf{t}_{1}$ which may be significantly modified by the presence of the liquid interface. Note that eq 9 is independent of the orientation angle of the particle axis; we have discarded a weak dependence on the contact angle. In the framework of this model, the deviations $\Delta V$ and $\Delta \omega$ are determined by the scale of the slip velocity $\mathbf{v}_{0}$ and the parameter $\epsilon$ that provides a measure for the nonuniform surface properties.

In Figure 10, we show, for each value of peroxide concentration, measured values of $V$ and $\Delta V$, and the corresponding quantities of the vertical component of the angular velocity $\omega$. Remarkably, the standard deviation $\Delta V$ is close to $R \Delta \omega$, where $R$ is the particle radius and $\Delta \omega$ the root mean square of the angular velocity. Indeed, with the particle

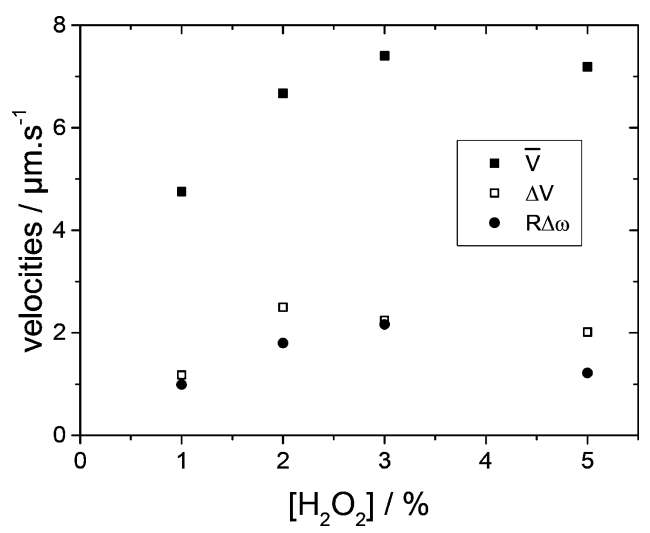

Figure 10. Concentration dependence of the mean velocity $V$ and of the standard deviation $\Delta V$. In order to highlight the strong correlation of $\Delta V$ and the root mean square of the angular velocity $\Delta \omega$, we multiply the latter with the particle radius $R$. Note that, because of the equal probability of left and right turning particles, the average angular velocity vanishes, $\omega=0$. Note that Figure 9 shows absolute values $\omega=$ $|\omega|$. 
radius $R=1.06 \mu \mathrm{m}$ one finds that the above theoretical expressions satisfy the relation

$$
\frac{\Delta V}{R \Delta \omega} \cong 1
$$

which agrees rather well with the measured values.

The main assumptions of our model (eq 8) are that the deviations of the slip velocity are short range and isotropic. The agreement of eq 10 with the experiment suggests that these assumptions are justified. In physical terms, this means that parallel and perpendicular fluctuations, as expressed by $\epsilon_{1}$ and $\epsilon_{2}$, have similar statistical properties. In other words, the nonuniform surface properties result in a random contribution to the slip velocity which is isotropic.

Within our model, the deviations $V-V$ and $\omega-\omega$ are uncorrelated, since their sign and magnitude are determined by different modes of the inhomogeneous surface properties; the data of Figure 9B agree qualitatively with this statement.

From the model (eq 8) one would expect that the inhomogeneity parameter $\epsilon$ is independent of the activity. The experimental data for $c=1 \%, 2 \%, 3 \%, 5 \%$ result in the values $\epsilon=0.15,0.2,0.19,0.15$, which at least qualitatively agree with what is expected from theory. Together with Figure 10, these numbers suggest that our simple model grasps the main features of the nonuniform surface properties of real Janus particles. These results provide evidence that each particle shows a slightly different asymmetry. These differences in the fabrication produce velocities $V$ and $\omega$ which are only weakly correlated but which show similar mean square deviations. Moreover, coating thickness and shape seem to have an impact on the velocities comparable to the impact of the $\mathrm{H}_{2} \mathrm{O}_{2}$ concentration. $^{19,20}$

The above relations 5-7 would suggest that both $V$ and $\omega$ increase linearly with the $\mathrm{H}_{2} \mathrm{O}_{2}$ fuel concentration. For the velocity $V$, this behavior was indeed observed previously. ${ }^{4}$ The present study, on the contrary, reveals quite a different behavior: data of Figure 10 increase only weakly at the lowest concentrations and are roughly constant at higher fuel concentration. The three quantities $V, \Delta V, \Delta \omega$ show a similar variation with concentration, which supports our simple model in terms of fluctuations of the slip velocity (8).

Increase of the Rotational Diffusion at High Angular Velocities. Contact line pinning and jumps between nearby metastable states are well known phenomena for fluid interfaces at a solid surface. For passive bare spherical colloids, we have recently shown that contact line fluctuations lead to additional dissipations in the translational motion. ${ }^{33}$ Within the model proposed by Boniello et al., a slowing down of $D_{\mathrm{r}, \|}$ is not expected for perfectly spherical bare particles. ${ }^{33}$

What we do expect, however, is that contact line jumps result in time dependent fluctuations of the slip velocity, which in turn increases the effective rotational diffusion $D_{\mathrm{r}, \|}$. Indeed, nanometric jumps ${ }^{33}$ of the contact line may locally modify the slip velocity at the particle surface and thus induce temporal fluctuations of the velocities, $V=V_{0}+\delta V$ and $\omega=\omega_{0}+\delta \omega$. Given that the characteristic fluctuation time $\tau$ of the contact line jumps is shorter than the experimental time scale, ${ }^{33}$ the angular mean square displacement becomes

$$
\left\langle\Delta \varphi(t)^{2}\right\rangle=\omega_{0}^{2} \Delta t^{2}+2\left(D_{\mathrm{r}, \|}+\delta \omega^{2} \tau\right) \Delta t
$$

Hence, an additional contribution should be accounted in the linear term of the MSAD (see eq 1 ). Note that an increase of the effective rotational diffusion due to velocity fluctuations in eccentric microswimmers in the bulk has been recently discussed by Debnath et al. ${ }^{29}$ In eq 11 , the additional contribution to the linear term of the MSAD contains $\delta \omega^{2}$, which in a good approximation increases with the square of $\omega$. This increase could explain the trend of the data of Figure 7.

The trajectories of Figure $2 \mathrm{~A}$ show rotational motion that persists over a large number of periods. This implies that the temporal velocity fluctuations $\delta V$ and $\delta \omega$ of a given particle are significantly smaller than the structural differences $\Delta V$ and $\Delta \omega$ from one particle to another.

\section{SUMMARY AND CONCLUSIONS}

In this article, we have shown that the active motion of Janus platinum-silica colloids fabricated starting from spherical particles can deviate significantly from the rectilinear motion randomized by the Brownian rotational motion. Some circular like trajectories can always be observed in bulk or at the interface because of a break in the particle symmetry due to the variability of shapes of the Platinum coating. At the air-water interface, the severe slowing down of the rotational diffusion $D_{\mathrm{r}, \perp}$ perpendicular to the interfacial normal (see Figure 4) prevents the particle from spinning about the motion direction, and the trajectories reported here at the interface always keep the same rotation direction and appear much more regular. A slowing down of the rotational diffusion $D_{\mathrm{r}, \|}$ parallel to the interfacial normal was also observed at low velocities. These results have been discussed and modeled accounting for static inhomogeneities of the particle surface and velocity fluctuations due to jumps of the contact line between metastable pinning states. In order to shed some light into the severe slowing down of the rotational diffusion, we are currently investigating the impact of contact line pinning and partial wetting dynamics for passive Janus colloids at liquid interfaces.

\section{AUTHOR INFORMATION}

\section{Corresponding Author}

*E mail: antonio.stocco@umontpellier.fr.

ORCID *

Christophe Blanc: $000000028470478 \mathrm{X}$

Antonio Stocco: 0000000255244323

Notes

The authors declare no competing financial interest.

\section{ACKNOWLEDGMENTS}

Financial supports from ANR SURFANICOL ANR 14 CE07 0039 01, Labex Chemisyst and Labex Numev are acknowl edged. A.W. acknowledges financial support from Agence nationale de la recherche through contract ANR 13 IS04 0003.

\section{REFERENCES}

(1) Elgeti, J.; Winkler, R. G.; Gompper, G. Physics of Micro swimmers-single Particle Motion and Collective Behavior: A Review. Rep. Prog. Phys. 2015, 78 (5), 56601.

(2) Bechinger, C.; Di Leonardo, R.; Löwen, H.; Reichhardt, C.; Volpe, G.; Volpe, G. Active Particles in Complex and Crowded Environments. Rev. Mod. Phys. 2016, 88 (4), 1 DOI: 10.1103/ RevModPhys.88.045006.

(3) Kümmel, F.; ten Hagen, B.; Wittkowski, R.; Buttinoni, I.; Eichhorn, R.; Volpe, G.; Löwen, H.; Bechinger, C. Circular Motion of Asymmetric Self Propelling Particles. Phys. Rev. Lett. 2013, 110 (19), 198302. 
(4) Wang, X.; In, M.; Blanc, C.; Nobili, M.; Stocco, A. Enhanced Active Motion of Janus Colloids at the Water Surface. Soft Matter 2015, 11, 7376-7384.

(5) Baraban, L.; Tasinkevych, M.; Popescu, M. N.; Sanchez, S.; Dietrich, S.; Schmidt, O. G. Transport of Cargo by Catalytic Janus Micro Motors. Soft Matter 2012, 8 (1), 48.

(6) Van Teeffelen, S.; Löwen, H. Dynamics of a Brownian Circle Swimmer. Phys. Rev. E Stat. Nonlinear, Soft Matter Phys. 2008, 78 (2), $2-5$.

(7) Dietrich, K.; Renggli, D.; Zanini, M.; Volpe, G.; Buttinoni, I.; Isa, L. Two Dimensional Nature of the Active Brownian Motion of Catalytic Microswimmers at Solid and Liquid Interfaces. New J. Phys. 2017, 19 (6), 65008.

(8) Marine, N. A.; Wheat, P. M.; Ault, J.; Posner, J. D. Diffusive Behaviors of Circle Swimming Motors. Phys. Rev. E Stat. Nonlinear, Soft Matter Phys. 2013, 87 (5), 1-10.

(9) Walther, A.; Müller, A. H. E. Janus Particles. Soft Matter 2008, 4 (4), 663.

(10) Jiang, S.; Chen, Q.; Tripathy, M.; Luijten, E.; Schweizer, K. S.; Granick, S. Janus Particle Synthesis and Assembly. Adv. Mater. 2010, 22, 1060-1071.

(11) Ondarçuhu, T.; Fabre, P.; Raphaël, E.; Veyssié, M. Specific Properties of Amphiphilic Particles at Fluid Interfaces. J. Phys. (Paris) 1990, 51, 1527-1536.

(12) Wang, W.; Duan, W.; Ahmed, S.; Mallouk, T. E.; Sen, A. Small Power: Autonomous Nano and Micromotors Propelled by Self Generated Gradients. Nano Today 2013, 8 (5), 531-534.

(13) Soler, L.; Magdanz, V.; Fomin, V. M.; Sanchez, S.; Schmidt, O. G. Self Propelled Micromotors for Cleaning Polluted Water. ACS Nano 2013, 7 (11), 9611-9620.

(14) Jurado Sánchez, B.; Sattayasamitsathit, S.; Gao, W.; Santos, L.; Fedorak, Y.; Singh, V. V.; Orozco, J.; Galarnyk, M.; Wang, J. Self Propelled Activated Carbon Janus Micromotors for Efficient Water Purification. Small 2015, 11 (4), 499-506.

(15) Howse, J.; Jones, R.; Ryan, A.; Gough, T.; Vafabakhsh, R.; Golestanian, R. Self Motile Colloidal Particles: From Directed Propulsion to Random Walk. Phys. Rev. Lett. 2007, 99 (4), 48102.

(16) Ebbens, S.; Gregory, D. a. DA; Dunderdale, G.; Howse, J. R.; Ibrahim, Y.; Liverpool, T. B.; Golestanian, R. Electrokinetic Effects in Catalytic Platinum Insulator Janus Swimmers. EPL (Europhysics Lett. 2014, 106, 58003.

(17) Choudhury, U.; Soler, L.; Gibbs, J. G.; Sanchez, S.; Fischer, P. Surface Roughness Induced Speed Increase for Active Janus Micro motors. Chem. Commun. 2015, 51 (41), 8660-8663.

(18) Gibbs, J. G.; Zhao, Y. P. Autonomously Motile Catalytic Nanomotors by Bubble Propulsion. Appl. Phys. Lett. 2009, 94 (16), 163104.

(19) Gregory, D. a.; Campbell, A. I.; Ebbens, S. J. The Effect of Catalyst Distribution on Spherical Bubble Swimmer Trajectories. J. Phys. Chem. C 2015, 119, 15339-15348.

(20) Archer, R. J.; Campbell, A. I.; Ebbens, S. Glancing Angle Metal Evaporation Synthesis of Catalytic Swimming Janus Colloids with Well Defined Angular Velocity. Soft Matter 2015, 11, 6872-6880.

(21) Pawar, A. B.; Kretzschmar, I. Multifunctional Patchy Particles by Glancing Angle Deposition. Langmuir 2009, 25 (16), 9057-9063.

(22) Gibbs, J. G.; Zhao, Y. P. Design and Characterization of Rotational Multicomponent Catalytic Nanomotors. Small 2009, 5 (20), 2304-2308.

(23) He, Y.; Wu, J.; Zhao, Y. Designing Catalytic Nanomotors by Dynamic Shadowing Growth. Nano Lett. 2007, 7 (5), 1369-1375.

(24) Nourhani, A.; Ebbens, S. J.; Gibbs, J. G.; Lammert, P. E. Spiral Diffusion of Rotating Self Propellers with Stochastic Perturbation. Phys. Rev. E: Stat. Phys., Plasmas, Fluids, Relat. Interdiscip. Top. 2016, 94, 1-5.

(25) Girot, A.; Danné, N.; Würger, A.; Bickel, T.; Ren, F.; Loudet, J. C.; Pouligny, B. Motion of Optically Heated Spheres at the Water Air Interface. Langmuir 2016, 32 (11), 2687-2697.
(26) Moyses, H.; Palacci, J.; Sacanna, S.; Grier, D. G. Trochoidal Trajectories of Self Propelled Janus Particles in a Diverging Laser Beam. Soft Matter 2016, 12 (30), 6357-6364.

(27) Wang, X.; In, M.; Blanc, C.; Malgaretti, P.; Nobili, M.; Stocco, A. Wetting and Orientation of Catalytic Janus Colloids at the Surface of Water. Faraday Discuss. 2016, 191, 305-324.

(28) Love, J. C.; Gates, B. D.; Wolfe, D. B.; Paul, K. E.; Whitesides, G. M. Fabrication and Wetting Properties of Metallic Half Shells with Submicron Diameters. Nano Lett. 2002, 2 (8), 891-894.

(29) Debnath, D.; Ghosh, P. K.; Li, Y.; Marchesoni, F.; Li, B. Diffusion of Eccentric Microswimmers. Soft Matter 2016, 12, 20172024.

(30) Ebbens, S. J.; Howse, J. R. Direct Observation of the Direction of Motion for Spherical Catalytic Swimmers. Langmuir 2011, 27 (20), 12293-12296.

(31) Ebbens, S.; Jones, R. A. L.; Ryan, A. J.; Golestanian, R.; Howse, J. R. Self Assembled Autonomous Runners and Tumblers. Phys. Rev. E Stat. Nonlinear, Soft Matter Phys. 2010, 82, 1 DOI: 10.1103/ PhysRevE.82.015304.

(32) O’Neill, M. E.; Ranger, K. B.; Brenner, H. Slip at the Surface of a Translating-rotating Sphere Bisected by a Free Surface Bounding a Semi Infinite Viscous Fluid: Removal of the Contact Line Singularity. Phys. Fluids 1986, 29 (4), 913.

(33) Boniello, G.; Blanc, C.; Fedorenko, D.; Medfai, M.; Mbarek, N. B.; In, M.; Gross, M.; Stocco, A.; Nobili, M. Brownian Diffusion of a Partially Wetted Colloid. Nat. Mater. 2015, 14 (9), 908-911.

(34) Boniello, G.; Stocco, A.; Gross, M.; In, M.; Blanc, C.; Nobili, M. Translational Viscous Drags of an Ellipsoid Straddling an Interface between Two Fluids. Phys. Rev. E: Stat. Phys., Plasmas, Fluids, Relat. Interdiscip. Top. 2016, 94 (1), 12602.

(35) Bian, X.; Kim, C.; Karniadakis, G. E. 111 Years of Brownian Motion. Soft Matter 2016, 12 (30), 6331-6346.

(36) Philipse, A. P. Notes on Brownian Motion; 2011; http:// userpages.umbc.edu/ dfrey1/ench630/philipse.

(37) Bickel, T.; Zecua, G.; Würger, A. Polarization of Active Janus Particles. Phys. Rev. E Stat. Nonlinear, Soft Matter Phys. 2014, 89 (5), 1 DOI: 10.1103/PhysRevE.89.050303. 